\title{
Image-guided Biopsy of Mesenteric, Omental, and Peritoneal Disease
}

\author{
Joshua Cornman-Homonoff, MD ${ }^{1}$ David C. Madoff, MD ${ }^{1}$ \\ ${ }^{1}$ Division of Interventional Radiology, Department of Radiology, NewYork- \\ Presbyterian Hospital/Weill Cornell Medical Center, New York, New York \\ Address for correspondence David C. Madoff, MD, Division of \\ Interventional Radiology, Department of Radiology, NewYork-Presbyterian \\ Hospital/Weill Cornell Medical Center, 525 East 68th Street P-518, \\ Dig Dis Interv 2018;2:106-115. \\ New York, NY 10065 (e-mail: dcm9006@med.cornell.edu).
}

Abstract
Keywords
- image-guided biopsy
- peritoneal disease
- mesentery
- omentum

The peritoneum, omenta, and mesenteries can be affected by a myriad of disease processes, but many common pathologies cannot be definitively distinguished based on clinical history and imaging characteristics alone. Percutaneous image-guided biopsy is a safe, well-tolerated procedure with high diagnostic accuracy, which has supplanted more invasive means of obtaining tissue and is increasingly essential in directing patient care. An understanding of the indications, pre-procedural evaluation, technical considerations, and potential complications is essential for the radiologist who performs these procedures, and more broadly for any clinician who may request them.

The peritoneum and adjacent spaces are anatomically complex and can be affected by a myriad of disease processes. Crosssectional imaging is the mainstay of evaluation, but many common pathologies cannot be definitively distinguished based on clinical history and imaging characteristics alone. In cases of diagnostic uncertainty, tissue sampling represents the best option for evaluation of disease pathology and extent. Additionally, in the era of personalized medicine, obtaining tissue for genetic and molecular analysis will become increasingly essential for directing cancer therapy and assessing response.

Percutaneous image-guided biopsy is a minimally invasive, safe, effective, and proven technique, which has been applied throughout the body for tissue sampling. As its role in patient management is only likely to expand, an understanding of the unique considerations for application within the peritoneum, omenta, and mesenteries is essential for any radiologist who may perform this procedure. This article provides an abbreviated review of the relevant anatomy and pathology, considerations in patient selection and evaluation, and details regarding the technical factors, which affect rates of success and complication.

\section{Anatomic Considerations}

\section{Anatomy}

The abdomen and pelvis are comprised of two distinct anatomic spaces, the peritoneal cavity and the subperitoneal

received

March 12, 2018

accepted after revision

April 20, 2018

published online

June 8, 2018 space, which are separated by a continuous layer of mesothelium termed the peritoneum. ${ }^{1}$ The peritoneal cavity is a potential space, which extends through the abdomen and pelvis. It contains no organs and only a small amount of fluid ( $100 \mathrm{~mL}$ ), which lubricates the visceral and parietal peritoneum as they slide over each other. ${ }^{2}$ It is divided into the greater and lesser sacs, which communicate via the epiploic foramen, as well as smaller subdivisions resulting from peritoneal infoldings. The term subperitoneal space refers to the continuous subserosal areolar tissue that lines the surfaces of the peritoneum and includes all mesenteries, peritoneal ligaments, and suspended organs. ${ }^{3}$ All abdominopelvic organs and their supporting structures are thus considered to be within this space. ${ }^{1}$

A mesentery is a double layer of peritoneum separated by connective tissue and fat. Mesenteries suspend the organs for which they are named and transmit blood vessels, lymphatics, and nerves to and from the body wall. ${ }^{2}$ An omentum is a double-layered fold of peritoneum that attaches to the stomach. The greater omentum hangs freely from the greater curvature into the lower abdomen before folding upon itself to attach to the transverse colon, resulting in a four-layered structure; the lesser omentum extends between the lesser curvature and the liver, separating the greater and lesser sacs (-Fig. 1). Peritoneal ligaments connect organs to other organs or to the abdominal wall. ${ }^{2}$ There are 11 such peritoneal reflections in the upper abdomen, including the two
Copyright $\odot 2018$ by Thieme Medical Publishers, Inc., 333 Seventh Avenue, New York, NY 10001, USA. Tel: +1(212) 584-4662.
Issue Theme Intra-abdominal Fluid Collections and Biopsies; Abdominal Trauma; Guest Editor, Sanjay Gupta, MD
DOI https://doi.org/ 10.1055/s-0038-1660499. ISSN 2472-8721. 

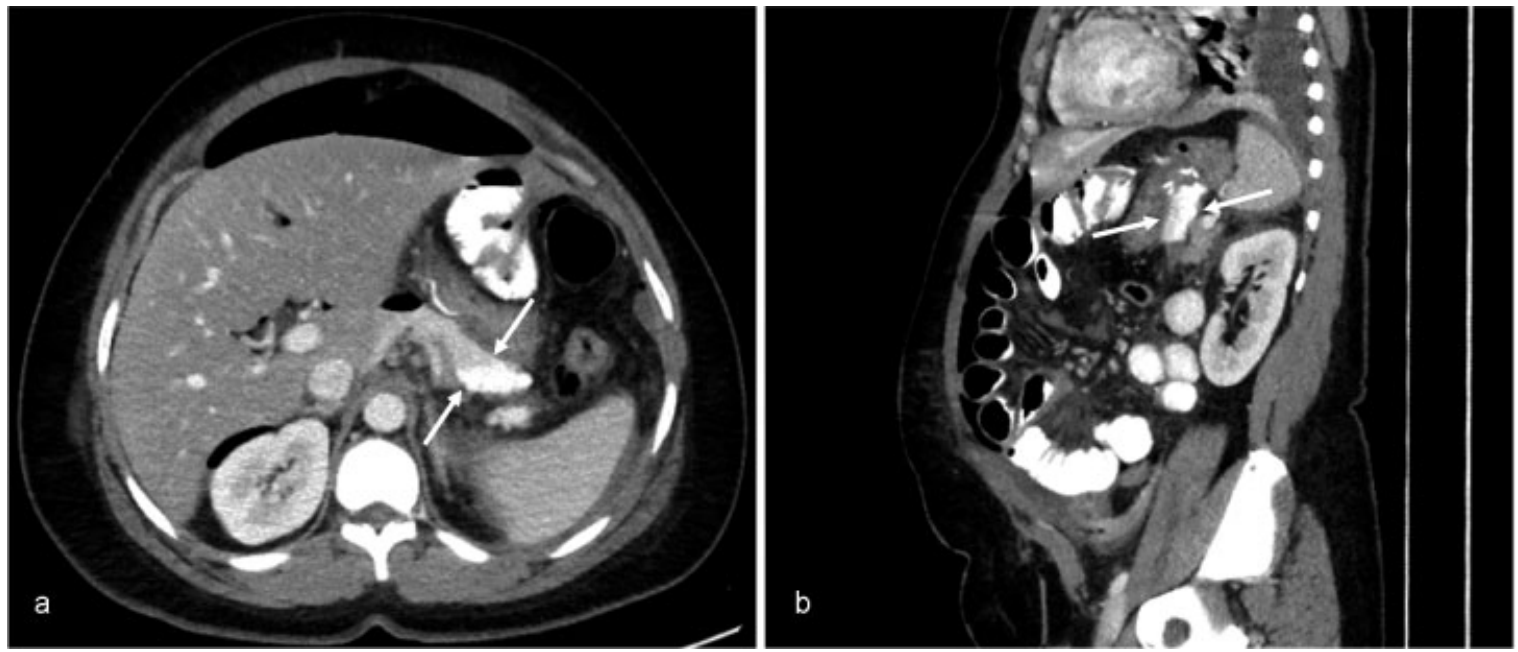

Fig. 1 A 37-year-old male on postoperative day 1 from gastric bypass with concern for anastomotic leak. (A) Axial and (B) sagittal images from an oral and intravenous contrast-enhanced CT demonstrate extravasated oral contrast material collecting in and distending the lesser sac (arrows). $\mathrm{CT}$, computed tomography.

lesser omental subdivisions: the gastrohepatic ligament, a membranous double-layered structure connecting the liver and stomach, and the hepatoduodenal ligament, the thickened free edge of the lesser omentum extending between the duodenum and liver, which encases the portal vein, hepatic artery, and bile duct. ${ }^{4}$ Together, the various peritoneal folds combine to divide the peritoneal cavity into multiple recesses and fossae, which determine the direction and extent of intraperitoneal disease spread. ${ }^{5}$

\section{Pathways of Disease Spread}

Understanding the mechanisms and pathways of disease spread throughout the abdominopelvic cavity can assist the radiologist in both determining the origin of the disease process and in predicting the likely direction of progression. In general, malignancies spread via direct extension, intraperitoneal seeding, lymphatic dissemination, or hematogenous dispersion. ${ }^{6,7}$ All of these pathways are in effect within the peritoneum and adjacent spaces, but special consideration should be given to peritoneal, subperitoneal, and transperitoneal pathways, which are comparatively unique. ${ }^{4}$

Peritoneal dissemination involves confined spread within the peritoneal cavity and may manifest as tumor implants, abscesses, ascites, or pneumoperitoneum. In cases of focal disease, peritoneal anatomy helps explain the direction and extent of spread. For example, disease may be confined to either the supramesocolic or inframesocolic spaces, which are separated by the transverse mesocolon, or further into the right or left divisions of the supramesocolic space, which are divided by the falciform ligament. ${ }^{7}$ Subperitoneal dissemination refers to the spread throughout the continuous subperitoneal space. Given its extensive and interconnected nature, disease may extend in many directions including into the retroperitoneum, mesentery, peritoneal ligaments, and/ or under the peritoneal surface of organs. Transperitoneal spread occurs when disease traverses the thin peritoneum, which consists of a single layer of mesothelial cells. ${ }^{1}$ Though this may happen in either direction, it occurs most commonly when subperitoneal disease extends to involve the peritoneal cavity.

\section{Pathology}

The identification of pathology affecting the peritoneum and adjacent spaces may occur incidentally or in the setting of directed investigation. The potential abnormalities are numerous given the complexity, centralization, and interconnectedness of the anatomy, but in the setting of the current discussion, the most common biopsy targets are mesenteric masses and lymphadenopathy.

Mesenteric masses should initially be classified by whether they are solid or cystic, as solid masses are usually neoplastic, whereas entirely cystic lesions are typically benign. ${ }^{8}$ The major exception to this is sclerosing mesenteritis, which manifests as a solid mass, but is without malignant potential. The most common solid masses to affect the mesentery are metastases and lymphoma, which are much more common than are primary tumors. ${ }^{8,9}$ Metastases resulting from direct extension are most commonly those of pancreatic and gastric origin, whereas peritoneal and omental metastases ("carcinomatosis") most commonly arise from ovarian, gastric, colorectal, and pancreatic cancers. $^{10-12}$ Peritoneal spread is often accompanied by the development of malignant ascites, and the flow of this fluid into the dependent portions of the peritoneum commonly results in the earliest metastatic seeding occurring in the right lower quadrant mesentery or rectouterine pouch (-Fig. 2). ${ }^{9}$

Though primary mesenteric neoplasms are rare, many different tumors may develop from the large variety of tissue types present. Among the various cell lines are those comprising the peritoneal surfaces, connective and fatty tissues, nerves, lymphatic structures, and blood vessels. ${ }^{13}$ Benign tumors, including lipomas, cystic lymphangiomas, and desmoid tumors, and malignant tumors, such as liposarcomas, 

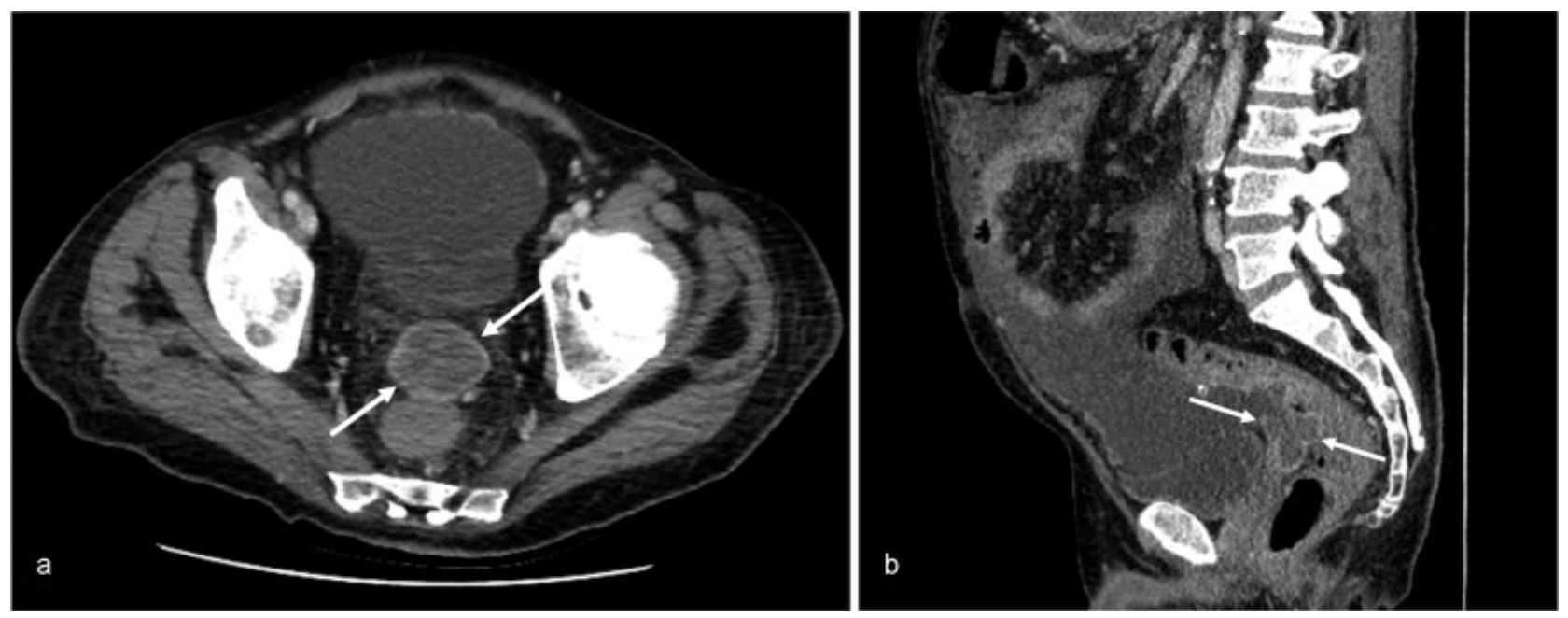

Fig. 2 A 58-year-old male with pancreatic adenocarcinoma metastatic to the liver and peritoneum. (A) Axial and (B) sagittal images from a contrast-enhanced CT demonstrate a hypoattenuating peritoneal implant in the rectouterine pouch of Douglas (arrows). CT, computed tomography.

leiomyosarcomas, malignant fibrous histiocytomas, lipoblastomas, and lymphangiosarcomas, have all been described. ${ }^{14}$ Primary peritoneal tumors include malignant peritoneal mesothelioma, which is associated with asbestos exposure and occurs far less commonly in the peritoneum than in the pleura or pericardium, benign cystic mesothelioma, which affects young women and has no relation to asbestos exposure, and desmoplastic small round cell tumor, which affects predominantly children and young adults. ${ }^{6,13}$

Improved scanning techniques have resulted in increased spatial resolution, decreased volume averaging, and reduced motion artifact, leading to more frequent identification of "normal" mesenteric lymph nodes. In one study examining CTscans in trauma patients without known underlying cause for mesenteric lymphadenopathy, 47 (39\%) of 120 patients were found to have mesenteric lymph nodes measuring over $3 \mathrm{~mm}$ in short axis. ${ }^{15}$ As such, differentiation between normal and pathologic nodes based on imaging characteristics is difficult but is necessary to minimize unnecessary biopsy. Although size is the most commonly utilized criterion, additional findings should be incorporated into the interpretive algorithm, including distribution, computed tomography (CT) attenuation/magnetic resonance (MR) intensity, and contrast enhancement; clinical history too is essential.

Broadly speaking, mesenteric adenopathy may result from malignancy, inflammation, and/or infection. Although almost any malignancy can involve mesenteric nodes, lymphoma is the most common. ${ }^{16}$ It is a soft tumor, which tends to grow around and displace normal structures, and classically homogenously enhances and only calcifies after treatment. The most common sources of mesenteric nodal metastases include cancers of the breast, lung, pancreas, and gastrointestinal tract. ${ }^{16}$ Gastrointestinal carcinoid tumors, though overall uncommon, metastasize to the mesentery in 40 to $80 \%$ of cases. ${ }^{17}$ Inflammatory causes of mesenteric lymphadenopathy include local processes, such as appendicitis, cholecystitis, and diverticulitis, more generalized conditions, such as inflammatory bowel disease and mesenteric panniculitis, and systemic diseases including systemic lupus erythematosus, systemic sclerosis, rheumatoid arthritis, and sarcoidosis. ${ }^{18-20}$ These conditions are generally easily distinguished from malignancy based on the clinical picture and additional imaging findings. Infectious etiologies include mesenteric adenitis, Yersinia enterocolitica, human immunodeficiency virus (HIV) (either primarily or more commonly secondary to an opportunistic infection), tuberculosis, and Whipple disease. ${ }^{21-23}$

\section{Patient Considerations}

\section{Indications}

Percutaneous biopsy is associated with lower morbidity and mortality than open or excisional biopsy and consequently should usually be the initial approach for tissue sampling. ${ }^{24}$ Biopsy is most commonly utilized to confirm suspected malignancy, obtain tissue for microbiologic analysis, stage patients with known cancer, and determine whether residual masses following treatment represent viable tumor or necrotic tissue; in cases in which clinical suspicion and biopsy results are discordant, repeat biopsy may be necessary. As continued evolution occurs within the field of personalized medicine, obtaining tissue for genetic and molecular characterization will comprise an increasingly substantial percentage of biopsy indications. ${ }^{25}$

Percutaneous biopsy should be considered when clinical history, physical examination, laboratory evaluation, and imaging studies are insufficient to arrive at a diagnosis and no other non-invasive means of investigation are available. Positron emission tomography (PET)-CT, while useful in the detection of focally increased metabolic activity, is of limited utility in cases in which both malignant and inflammatory processes are in the differential or where tumors with a low metabolic rate are a consideration. ${ }^{26}$ Similarly, small tumors 
may escape detection when they are below the resolution of the study, and determination of the cellular origin of metastatic disease is limited by the inability to obtain tissue for direct histopathologic analysis. Cytologic evaluation of ascitic fluid obtained via paracentesis is minimally invasive, but diagnostically imperfect, with determination of site-specific tumor origin obtained in as few as $60 \%$ of cases. ${ }^{27}$

While there are no absolute contraindications to percutaneous biopsy, and patients should be evaluated on an individual basis, several relative contraindications have been identified. Among them are significant coagulopathy that cannot be adequately corrected, severely compromised cardiopulmonary function or hemodynamic instability, lack of safe access to the lesion, inability of the patient to cooperate with or to be positioned for the procedure, and pregnancy in cases when imaging guidance involves ionizing radiation. $^{24}$

\section{Patient Selection and Pre-procedural Evaluation}

The initial evaluation is standard for any interventional procedure, including review of clinical history, laboratory studies, and available imaging, and performance of a targeted physical examination. The need for biopsy should be readdressed, with consideration given to whether noninvasive studies or endoscopic biopsy would provide the needed information and obviate the risks associated with percutaneous access. ${ }^{28}$ In addition to determining necessity, the radiologist should assess technical feasibility and choose the safest possible approach. When multiple lesions are present, the lowest risk and the highest yield among them should be selected as the target; in patients with widespread lymphadenopathy, this may include very low risk and easily accessible nodes, such as in the axillary or inguinal regions. The shortest path of travel should be chosen, and traversal of major organs or vessels should be minimized or avoided where possible. If prone positioning or breath holding is expected to be necessary, patient cooperation should be assessed. If the patient cannot tolerate the needed positioning, cannot follow instructions, or has any of several medical comorbidities, which may limit their ability to tolerate moderate sedation (i.e., decompensated heart failure, extensive pulmonary disease, morbid obesity, sleep apnea, and pregnancy), anesthesia should be consulted.

As hemorrhage is the most common biopsy complication, and application of direct pressure is not possible with deep lesions, steps should be taken to minimize the likelihood of bleeding. Assessment of platelet levels, prothrombin time (PT), and activated partial thromboplastin time (aPTT) is reasonable, although there is increasing debate regarding the need for routine testing with a more selective, patientspecific approach appearing to gain favor. ${ }^{29}$ At our institution, platelet levels above $50 \times 10^{9} / \mathrm{L}$ and international normalized ratio (INR) below 1.6 are generally considered safe, as prior studies have demonstrated minimal risk of major post-biopsy bleeding when values are within these ranges. ${ }^{30}$ In cases in which the coagulation parameters fall outside of these limits, the decision to proceed is specific to the radiologist and clinical scenario. Laboratory results obtained with 30 days are generally acceptable, although more recent studies may be required on a case-by-case basis, particularly in patients at increased risk of bleeding such as those on anticoagulation, with known bleeding disorders, or with comorbidities that may affect coagulability (i.e., malnutrition, liver disease, and renal failure). Patients on anticoagulation or antiplatelet therapy should have their medications held in accordance with the Society of Interventional Radiology guidelines; in cases in which newer medications are without established recommendations, withholding the drug for a minimum five half-lives is generally considered sufficient. $^{31,32}$ If the risk of complete discontinuation is considered excessively high, the need for the procedure should be reevaluated, and if it is still felt to be indicated, the patient may be converted to unfractionated heparin (half-life $\sim 60$ to $90 \mathrm{~min}$ ) to be discontinued shortly before the procedure. ${ }^{33}$ Low-molecular weight heparin has a halflife two to four times that of unfractionated heparin, and patients receiving it should have one dose held prior to the procedure. ${ }^{33}$ Additional care should also be taken to avoid hemorrhage while undertaking biopsy of potentially hypervascular lesions, such as renal or thyroid metastases, paragangliomas, and hemangiopericytomas.

\section{Technical Considerations}

\section{Selection of Imaging Modality}

Most biopsies are performed under either ultrasound (US) or CT guidance, with MR relegated to a secondary role because of its high cost, limited availability, and long procedure times. Modality selection ultimately depends on radiologist preference and experience, but factors to consider include equipment availability, nature, size, and location of the target lesion; adequacy of lesion visualization; and cost. Additionally, patient comorbidities may affect the choice of modality. For example, patients who are unable to lay flat may not tolerate the positioning required during CT guidance, and elevated body mass index can make US visualization challenging, although it has been demonstrated that utilization of a transabdominal approach with compression of the abdominal wall frequently provides visualization sufficient to achieve successful biopsy. ${ }^{34}$

US guidance has numerous potential advantages compared with CT (-Fig. 3). ${ }^{28,34-36}$ It allows real-time visualization of the needle, target, and surrounding structures, and use of color Doppler imaging helps in identification of adjacent vessels and post-biopsy bleeding complications. The transducer can be manipulated into any imaging plane allowing for a more flexible, non-axial approach, and application of pressure can displace underlying structures thereby reducing the needle-path distance. Additional benefits include the lack of ionizing radiation, portability, shorter procedure time, and lower overall cost relative to $\mathrm{CT}$ guidance. 37,38

Limitations of US include the substantial operator dependence, as well as inability to penetrate air-filled structures, bone, or thick soft tissue. The effects of operator dependence can potentially be partially mitigated by the use of a needle 

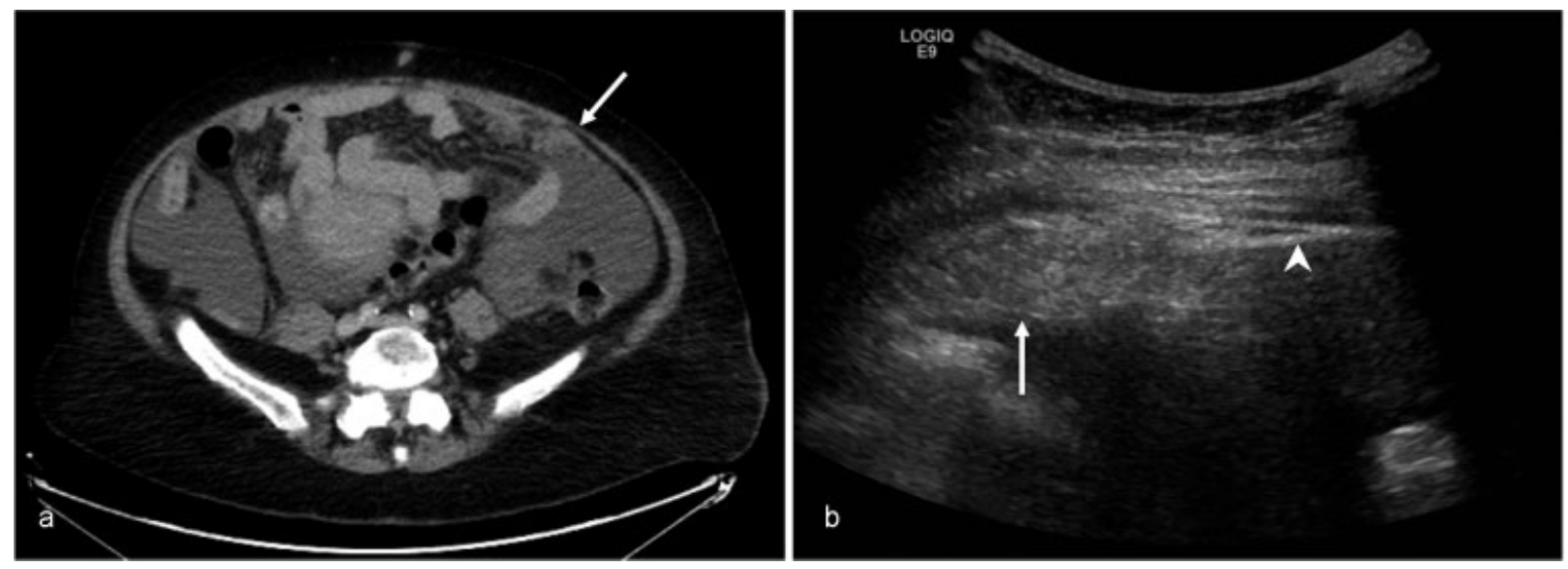

Fig. 3 A 67-year-old female with endometrial mass and peritoneal nodularity. (A) Axial contrast-enhanced CT image demonstrates large volume ascites with a nodule of soft tissue attenuation along the left anterior parietal peritoneum (arrow). (B) Supine axial US image obtained during biopsy demonstrates the biopsy needle (arrowhead) traversing the peritoneal implant (arrow). 22-gauge FNAB and 20-gauge CNB were performed via coaxial technique, confirming a diagnosis of metastatic endometrial carcinoma. CNB, core needle biopsy; CT, computed tomography; FNAB, fine-needle aspiration biopsy; US, ultrasound.

guide, which simplifies coordinated maneuvering of the needle and probe and has been suggested to improve biopsy yield and decrease complication rate when utilized by nonradiologists. ${ }^{39}$ Similarly, although CT has been favored over US for biopsy of masses deep in the mesentery and pelvis, particularly when small, multiple studies have demonstrated the feasibility and safety of targeting structures as small as $1 \mathrm{~cm}$ in such locations. ${ }^{34-36,40}$ For more superficial structures such as the omentum and peritoneum, US may be as good as or even superior to $\mathrm{CT}$ in terms of biopsy yield. ${ }^{41,42}$ Continued advancements in imaging technology, such as fusion of US images with CT or MR studies, usage of guidance software for more precise and rapid targeting, and utilization of US contrast material for improved tissue differentiation, will help in further increasing the accuracy and safety of biopsies performed under US guidance.

$\mathrm{CT}$ can be utilized to target lesions nearly anywhere in the body, including those not amenable to US guidance, and has traditionally been the favored guidance modality for biopsy of deep mesenteric and pelvic lesions (-Fig. 4). Unlike US, it provides high spatial and contrast resolution, a large field of view, and excellent delineation of the biopsy needle, target, and intervening soft tissues. It is not susceptible to interference from bowel gas or calcification, and the images obtained are easily correlated with prior functional imaging, such as $18 \mathrm{~F}$-fludeoxyglucose (FDG) PET, to ensure targeting of the appropriate area within larger, partially necrotic masses. Radiologists are also generally very familiar with CT images and, as a result, find CT-guided biopsy simpler and the learning curve shorter compared with US.

The main limitation of CT guidance is the absence of realtime imaging. This is partially mitigated by using CT fluoroscopy, which combines the high-resolution of conventional CT with the active imaging of fluoroscopy. It can be of particular benefit in targeting mobile lesions, such as those near the diaphragm or in restless patients. ${ }^{43}$ Usage of this technology decreases the delay between image acquisition and needle manipulation and has been shown to reduce procedure time relative to conventional CT guidance. ${ }^{38,44}$ The main concern is the potential for increased radiation dose, but with available techniques to reduce exposure (usage of needle holders to distance the operator's hands from the beam, placement of lead drapes, reduction in tube potential and current, usage of thinner image slices, and intermittent scanning during needle advancement), dosage can be maintained within acceptable levels. ${ }^{4-46}$

\section{Devices}

The selection of biopsy needle depends on the amount and composition of tissue necessary for diagnosis. Core needle biopsy (CNB) obtains a cylindrical tissue sample, which preserves architecture and allows for complete histologic analysis, whereas fine-needle aspiration biopsy (FNAB) collects individual cells for cytology, which can be rapidly evaluated but lack organization. FNAB may be performed through smaller needles, which cause less tissue trauma and, due to the rapidity of analysis, allows for intra-procedural determination of sample adequacy; however, due to the small amount of tissue obtained, only limited diagnostic testing can be performed. The techniques should be considered complimentary, and performance of both increases overall diagnostic sensitivity. ${ }^{47}$ In general, at our institution, FNAB is initially performed, and the sample is examined in real time to confirm needle position and presence of pathology. Following this, a variable number of core samples are obtained until sufficient tissue has been collected to allow for accurate histologic diagnosis.

Many needles are available which differ in length, caliber, tip configuration, and sampling mechanism. For example, CNB may be performed with either end-cutting or side-cutting devices, which have distinct attributes and benefits, and personal preference generally dictates which is selected. ${ }^{28}$ In general, CNB is performed with larger 14 to 20 -gauge needles, whereas FNAB is performed with smaller needles on the order of 20 to 25 gauge..$^{48} \mathrm{~A}$ modest association between needle size and risk of hemorrhage has been demonstrated, but use of 14- 

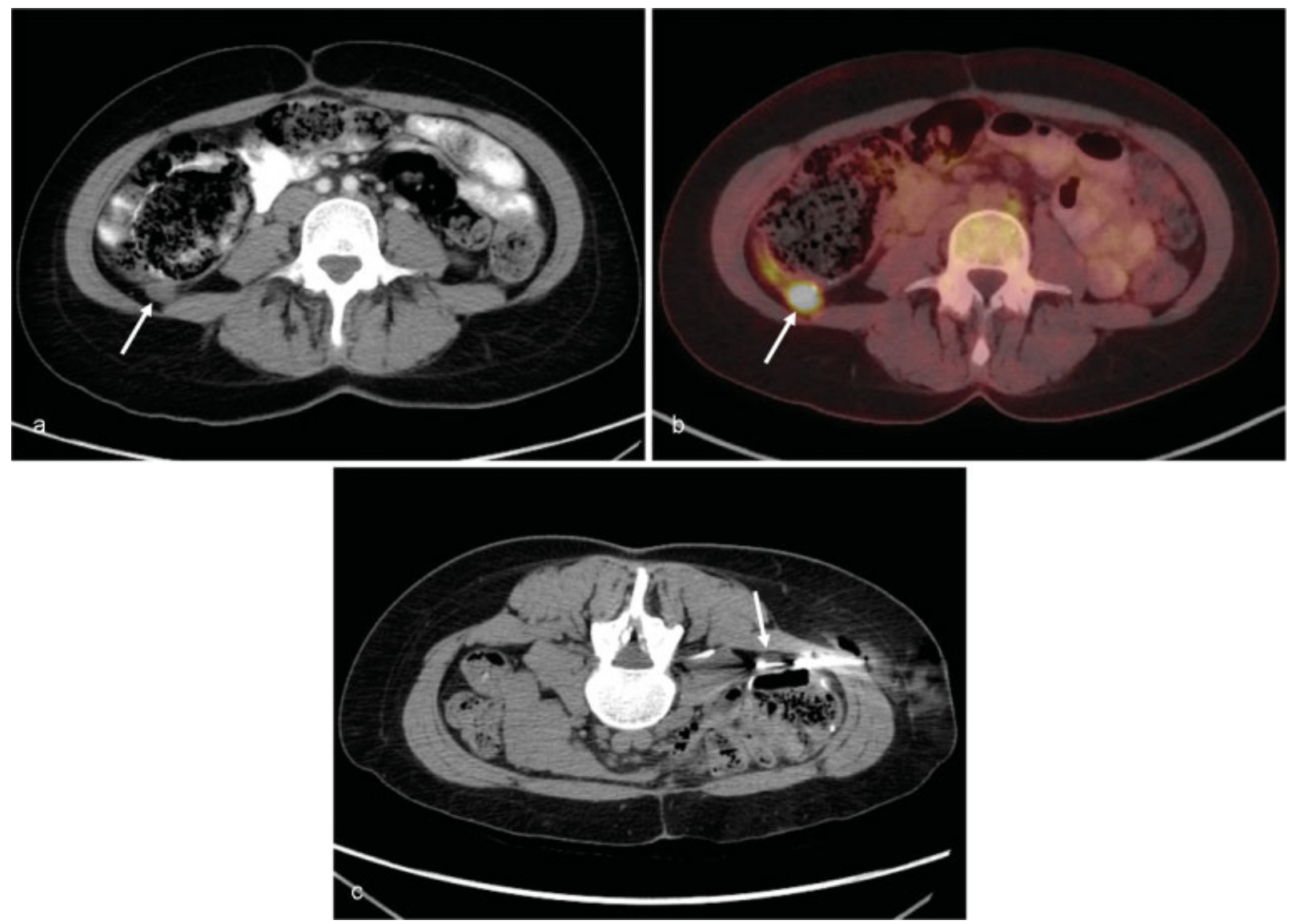

Fig. 4 A 40-year-old female with a history of colon cancer and concern for recurrence. (A) Axial contrast-enhanced CT image demonstrates a nodule of soft tissue attenuation in the right paracolic gutter (arrow). (B) Axial fused 18F-FDG-PET/CT image demonstrates associated hypermetabolism (arrow). (C) Prone CT-guided 20-gauge FNAB and 18-gauge CNB obtained via coaxial technique (arrow) confirmed recurrent disease. CNB, core needle biopsy; CT, computed tomography; FDG-PET, fludeoxyglucose-positron emission tomography; FNAB, fine-needle aspiration biopsy; US, ultrasound.

to 16-gauge cutting needles for peritoneal biopsy has been reported without complication. ${ }^{49,50}$ At our institution, for typical peritoneal and mesenteric biopsies, FNAB is performed using a 22-gauge needle, and a 20-gauge automated sidecutting device is used for CNB.

\section{Techniques}

At our institution, most biopsies are performed under conscious sedation, which improves patient comfort, anxiety, and cooperation; decreases procedure times; increases accuracy; and reduces the potential for injury. Patients are required to fast for 6 to 8 hours before the procedure to minimize the risk of aspiration. In those with Mallampati class IV anatomy, a history of sleep apnea, or an otherwise difficult airway, evaluation by anesthesia is generally indicated; alternatively, the procedure may be performed under local anesthesia (using lidocaine with epinephrine) if the patient is cooperative and amenable to the attempt.

Patient positioning is selected to optimize both access to the target lesion and patient comfort. Masses in the upper abdomen or adjacent to the liver dome may be difficult to reach secondary to the presence of intervening lung. Placement of the patient in the ipsilateral decubitus position can deflate the lung and displace it from the costophrenic sulcus, thereby decreasing the risk of lung traversal and consequent pneumothorax or empyema. ${ }^{51}$ Decubitus positioning can also be helpful in shifting lesions or altering the configuration of overlapping structures, resulting in the opening of new or improved access windows. ${ }^{52}$ Subcostal approaches are preferred over intercostal for the superior maneuverability and lower risk of injury to intercostal vessels that they provide. ${ }^{48}$ If an intercostal approach is needed, raising the patient's ipsilateral arm above their head opens the intercostal space and improves access, although care must be taken to avoid excessive and prolonger stretching which may cause injury to the brachial plexus. If CT guidance is to be used, a combination of variable patient positioning and angling of the gantry can be used to take an oblique, nonaxial approach and thereby access otherwise difficult to reach targets in the subphrenic or upper pelvic regions. ${ }^{52}$

An anterior approach is often chosen for biopsy of anterior peritoneal, omental, or mesenteric lesions, with the main limitation being the presence of intervening abdominal viscera. Fortunately, multiple techniques to displace these structures are available. Hydrodissection involves the instillation of fluid (usually, $0.9 \%$ saline) to mechanically shift structures and create new access routes (-Fig. 5). It has previously been shown to have a high rate of technical 

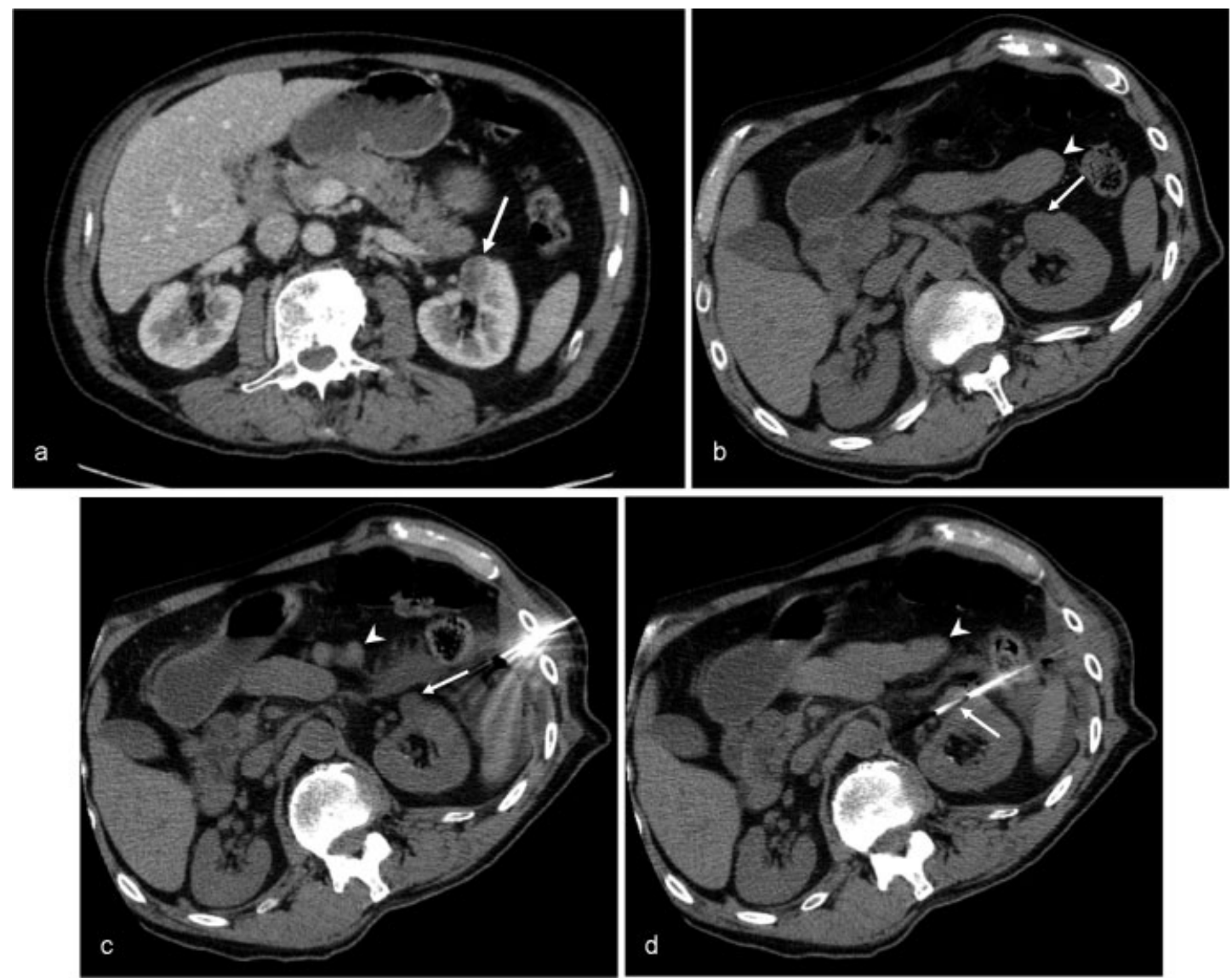

Fig. 5 A 68-year-old male with a left renal mass. (A) Axial contrast-enhanced CT image obtained in the nephrographic phase demonstrates a left renal cortical lesion (arrow). (B) Axial CT-guidance image obtained at procedure outset demonstrates bowel (arrowhead) in close proximity to the lesion (arrow). (C) After instillation of normal saline, the bowel loop (arrowhead) has been displaced and the lesion (arrow) is more easily accessible. (D) Biopsy (arrow) was performed without injury to the bowel (arrowhead), confirming a diagnosis of renal cell carcinoma.

success and may have the added benefit of improving the sonographic window during US-guided procedures. ${ }^{28,53,54}$ When US-guidance is utilized, manual pressure on the transducer can help to displace intervening bowel loops and mesentery, shorten the needle-path distance, improve visualization, and fix mobile masses. Of note, compression may collapse and thereby obscure blood vessels, leading some authors to recommend evaluation with color Doppler prior to the application of pressure to ensure that they are subsequently avoided. ${ }^{28}$ After initial soft tissue traversal, the use of a needle containing a blunt trocar tip can help to gently move intervening structures and decrease injury risk, as the needle is advanced toward the target. ${ }^{55,56}$

Organ transgression should only be undertaken when alternative approaches are not possible. Most solid organs, including the liver, spleen, and kidneys, can be safely traversed, although the path of travel should be as short as possible and selected to minimize the density of intervening neurovascular structures (i.e., avoid the porta hepatis and renal hila). ${ }^{28}$ Hollow organs are somewhat more variable in terms of puncture safety. The stomach is generally considered very low risk due to its thick wall and near sterility, with studies suggesting that needles as large as 18 -gauge may be introduced without fear of peritonitis or bleeding. ${ }^{57,58}$ The small bowel too is low risk, although smaller-gauge needles (20- to 22-gauge) are preferred. ${ }^{28}$ By contrast, all attempts should be made to avoid the colon due to its high bacterial content, risk of peritonitis should spillage occur, and potential for contamination of the biopsy specimen. ${ }^{59}$ If a transcolonic approach is necessary, the smallest possible needle should be utilized and as few passes as possible should be made. Consideration should also be given to pre-procedural bowel preparation and prophylactic antibiotic administration, although there is no evidence that the latter decreases complication rates.

A posterior approach may be needed for targets located at the base of the mesentery or which extend into the retroperitoneum. Such an approach usually involves passage through or alongside the quadratus lumborum and psoas muscles which, although technically feasible, makes trajectory correction more difficult. However, one potential advantage to the posterior approach is that should a hemorrhagic complication occur, the result would be a contained retroperitoneal hematoma which is prone to auto-tamponade rather than free intraperitoneal hemorrhage. ${ }^{35}$

Biopsies can be obtained with either coaxial or noncoaxial techniques. Deciding which to use depends in part upon the selected image guidance modality: the coaxial approach is commonly preferred for CT guidance, while direct puncture 
is often favored under US. Potential advantages of the coaxial technique include the following: the ability to obtain multiple samples without additional traversal of the overlying tissue, thereby reducing the likelihood of injury and patient discomfort; the ease and speed of collecting multiple samples, which reduces both procedure time and radiation exposure associated with CT-guided biopsies; the potential for tract embolization at the conclusion of the procedure; and decreased risk for tract seeding in cases of malignancy. ${ }^{48,60}$ The main disadvantages include usage of larger needles and prolonged needle dwell time, potentially resulting in tissue laceration secondary to respiratory motion. Despite these concerns, no difference in complication rate has been demonstrated between the techniques. ${ }^{61} \mathrm{~A}$ technical concern regarding the use of the coaxial technique is that, as the guide needle trajectory is essential unchanged between specimens, repeated sampling of the same intralesional site may make collection of additional tissue samples difficult. This can be partially mitigated using curved needles, which may result in increased tissue yield. ${ }^{62,63}$

\section{Outcomes}

Percutaneous image-guided biopsy of the peritoneum, omenta, and mesentery has been established as a safe, welltolerated procedure with high diagnostic accuracy. ${ }^{64}$ Complication rates are variable and dependent upon patient selection, biopsy site, and underlying pathology, but are almost invariably low. ${ }^{24}$ The two most commonly reported complications are puncture site pain and hemorrhage along the biopsy path. In the largest available series consisting of 186 biopsies of the omentum and peritoneum, Vadvala et al reported complications in five $(2.7 \%)$ patients, two of which were major and three minor, all in the mesenteric biopsy group..$^{65}$ One major complication consisted of an intra-abdominal abscess which formed following transcolonic biopsy and ultimately required laparotomy, left colectomy, and colostomy, and the other of a mesenteric hematoma resulting in a $4.0 \mathrm{~g} / \mathrm{dL}$ hemoglobin drop which was managed conservatively with admission and fluid resuscitation. All three minor complications were related to moderate to severe pain at the skin puncture site and were managed conservatively.

Other series report comparable rates of complication. In a series of 153 patient who underwent US-guided biopsy of the peritoneum or omentum, Wang et al encountered no serious complications; procedure site pain occurred in 15 patients and minor hemorrhage from the biopsy site in two, none of which required intervention. ${ }^{42}$ Hewitt et $\mathrm{al}^{41}$ reported a single rectus sheath hematoma after percutaneous CT- or US-guided biopsy in 149 women with peritoneal carcinomatosis, and Souza et $\mathrm{al}^{66}$ countered minor complications in three patients in their series of 111 peritoneal and omental mass biopsies. Although needle tract seeding has been reported, it is rare and was not observed by either Vadvala et al in 186 patients or Maturen et al in 128, possibly secondary to the consistent usage of a coaxial biopsy technique in both series. ${ }^{60,65,67-69}$

Although the diagnostic yield of percutaneous imageguided biopsy varies depending on technical and patient factors, multiple studies have confirmed its accuracy and reliability. In general, diagnostic yields on the order of 87 to 93\% have been reported regardless of guidance modality or biopsy site. ${ }^{41,50,66,70}$ More recently, Vadvala et al reported an overall sensitivity of $95.5 \%$ and specificity of $100 \%$ for CT- and US-guided omental and mesenteric biopsies, both of which were superior to prior studies and additionally noted that CNB had higher a diagnostic yield (98.4\%) compared to FNAB (84\%). ${ }^{65}$ Despite the latter finding, the authors recommended routinely obtaining both CNB and FNAB, as FNAB enhanced the diagnostic yield in a small number of cases in which core biopsy was unable to be performed secondary to potential procedure complications.

\section{Conclusion}

The peritoneum, omenta, and mesenteries can be affected by many disease processes, and percutaneous image-guided biopsy is a safe, well-tolerated procedure with high diagnostic accuracy which can be essential in distinguishing between them. A thorough understanding of the indications, workup, and technical considerations pertinent to the procedure will help the radiologist to achieve maximum diagnostic yield with the minimize number of complications regardless of the nature, size, or location of the biopsy target.

\section{Conflict of Interest}

None.

\section{References}

1 Pannu HK, Oliphant M. The subperitoneal space and peritoneal cavity: basic concepts. Abdom Imaging 2015;40(07):2710-2722

2 Moore KL, Dalley AF, Agur AMR. Clinically Oriented Anatomy. 7th ed. Philadelphia: Wolters Kluwer/Lippincott Williams \& Wilkins Health; 2014

3 Gore RM, Newmark GM, Thakrar KH, Mehta UK, Berlin JW. Pathways of abdominal tumour spread: the role of the subperitoneal space. Cancer Imaging 2009;9:112-120

4 Meyers MA, Oliphant M, Berne AS, Feldberg MA. The peritoneal ligaments and mesenteries: pathways of intraabdominal spread of disease. Radiology 1987;163(03):593-604

5 Kelley LL, Petersen CM. Sectional Anatomy for Imaging Professionals. 4th ed. St. Louis, Missouri: Elsevier; 2018

6 Robbins SL, Kumar V, Cotran RS. Robbins and Cotran Pathologic Basis of Disease. 8th ed. Philadelphia, PA: Saunders/Elsevier; 2010

7 Le O. Patterns of peritoneal spread of tumor in the abdomen and pelvis. World J Radiol 2013;5(03):106-112

8 Ezhapilli SR, Moreno CC, Small WC, Hanley K, Kitajima HD, Mittal PK. Mesenteric masses: approach to differential diagnosis at MRI with histopathologic correlation. J Magn Reson Imaging 2014;40 (04):753-769

9 Taffel MT, Khati NJ, Hai N, Yaghmai V, Nikolaidis P. De-misty-fying the mesentery: an algorithmic approach to neoplastic and nonneoplastic mesenteric abnormalities. Abdom Imaging 2014;39 (04):892-907

10 Okino Y, Kiyosue $\mathrm{H}$, Mori $\mathrm{H}$, et al. Root of the small-bowel mesentery: correlative anatomy and CT features of pathologic conditions. Radiographics 2001;21(06):1475-1490

11 Sadeghi B, Arvieux C, Glehen O, et al. Peritoneal carcinomatosis from non-gynecologic malignancies: results of the EVOCAPE 1 multicentric prospective study. Cancer 2000;88(02):358-363 
12 Winant AJ, Vora A, Ginter PS, Levine MS, Brylka DA. More than just metastases: a practical approach to solid mesenteric masses. Abdom Imaging 2014;39(03):605-621

13 Dufay C, Abdelli A, Le Pennec V, Chiche L. Mesenteric tumors: diagnosis and treatment. J Visc Surg 2012;149(04):e239-e251

14 Schwartz SI, Brunicardi FC. Schwartz's Principles of Surgery. 9th ed. New York: McGraw-Hill, Medical Pub. Division; 2010

15 Lucey BC, Stuhlfaut JW, Soto JA. Mesenteric lymph nodes: detection and significance on MDCT. AJR Am J Roentgenol 2005;184 (01):41-44

16 Lucey BC, Stuhlfaut JW, Soto JA. Mesenteric lymph nodes seen at imaging: causes and significance. Radiographics 2005;25(02): 351-365

17 Pantongrag-Brown L, Buetow PC, Carr NJ, Lichtenstein JE, Buck JL. Calcification and fibrosis in mesenteric carcinoid tumor: CT findings and pathologic correlation. AJR Am J Roentgenol 1995; 164(02):387-391

18 Rao PM, Rhea JT, Novelline RA. Sensitivity and specificity of the individual CT signs of appendicitis: experience with 200 helical appendiceal CT examinations. J Comput Assist Tomogr 1997;21 (05):686-692

19 Goldberg HI, Gore RM, Margulis AR, Moss AA, Baker EL. Computed tomography in the evaluation of Crohn disease. AJR Am J Roentgenol 1983;140(02):277-282

20 Calgüneri M, Oztürk MA, Ozbalkan Z, et al. Frequency of lymphadenopathy in rheumatoid arthritis and systemic lupus erythematosus. J Int Med Res 2003;31(04):345-349

21 Macari M, Hines J, Balthazar E, Megibow A. Mesenteric adenitis: CT diagnosis of primary versus secondary causes, incidence, and clinical significance in pediatric and adult patients. AJR Am J Roentgenol 2002;178(04):853-858

22 Radin R. HIV infection: analysis in 259 consecutive patients with abnormal abdominal CT findings. Radiology 1995;197(03): 712-722

23 Hulnick DH, Megibow AJ, Naidich DP, Hilton S, Cho KC, Balthazar EJ. Abdominal tuberculosis: CT evaluation. Radiology 1985;157 (01):199-204

24 Gupta S, Wallace MJ, Cardella JF, Kundu S, Miller DL, Rose SC; Society of Interventional Radiology Standards of Practice Committee. Quality improvement guidelines for percutaneous needle biopsy. J Vasc Interv Radiol 2010;21(07):969-975

25 Marshall D, Laberge JM, Firetag B, Miller T, Kerlan RK. The changing face of percutaneous image-guided biopsy: molecular profiling and genomic analysis in current practice. J Vasc Interv Radiol 2013;24(08):1094-1103

26 Fletcher JW, Djulbegovic B, Soares HP, et al. Recommendations on the use of 18F-FDG PET in oncology. J Nucl Med 2008;49(03): 480-508

27 Karoo RO, Lloyd TD, Garcea G, Redway HD, Robertson GS. How valuable is ascitic cytology in the detection and management of malignancy? Postgrad Med J 2003;79(931):292-294

28 Carberry GA, Lubner MG, Wells SA, Hinshaw JL. Percutaneous biopsy in the abdomen and pelvis: a step-by-step approach Abdom Radiol (NY) 2016;41(04):720-742

29 O'Connor SD, Taylor AJ, Williams EC, Winter TC. Coagulation concepts update. AJR Am J Roentgenol 2009;193(06):1656-1664

30 Atwell TD, Smith RL, Hesley GK, et al. Incidence of bleeding after 15,181 percutaneous biopsies and the role of aspirin. AJR Am J Roentgenol 2010;194(03):784-789

31 Patel IJ, Davidson JC, Nikolic B, et al; Standards of Practice Committee, with Cardiovascular and Interventional Radiological Society of Europe (CIRSE) Endorsement. Consensus guidelines for periprocedural management of coagulation status and hemostasis risk in percutaneous image-guided interventions. J Vasc Interv Radiol 2012;23(06):727-736

32 Patel IJ, Davidson JC, Nikolic B, et al; Standards of Practice Committee, with Cardiovascular and Interventional Radiological Society of Europe (CIRSE) Endorsement; Standards of Practice
Committee of the Society of Interventional Radiology. Addendum of newer anticoagulants to the SIR consensus guideline. J Vasc Interv Radiol 2013;24(05):641-645

33 Cook BW. Anticoagulation management. Semin Intervent Radiol 2010;27(04):360-367

34 Memel DS, Dodd GD III, Esola CC. Efficacy of sonography as a guidance technique for biopsy of abdominal, pelvic, and retroperitoneal lymph nodes. AJR Am J Roentgenol 1996;167(04): 957-962

35 Fisher AJ, Paulson EK, Sheafor DH, Simmons CM, Nelson RC. Small lymph nodes of the abdomen, pelvis, and retroperitoneum: usefulness of sonographically guided biopsy. Radiology 1997; 205(01):185-190

36 Gupta S, Rajak CL, Sood BP, Gulati M, Rajwanshi A, Suri S. Sonographically guided fine needle aspiration biopsy of abdominal lymph nodes: experience in 102 patients. J Ultrasound Med 1999;18(02):135-139

37 Sheafor DH, Paulson EK, Simmons CM, DeLong DM, Nelson RC. Abdominal percutaneous interventional procedures: comparison of CT and US guidance. Radiology 1998;207(03):705-710

38 Sheafor DH, Paulson EK, Kliewer MA, DeLong DM, Nelson RC. Comparison of sonographic and CT guidance techniques: does CT fluoroscopy decrease procedure time? AJR Am J Roentgenol 2000; 174(04):939-942

39 Prasad N, Kumar S, Manjunath R, et al. Real-time ultrasoundguided percutaneous renal biopsy with needle guide by nephrologists decreases post-biopsy complications. Clin Kidney J 2015;8 (02):151-156

40 Ho LM, Thomas J, Fine SA, Paulson EK. Usefulness of sonographic guidance during percutaneous biopsy of mesenteric masses. AJR Am J Roentgenol 2003;180(06):1563-1566

41 Hewitt MJ, Anderson K, Hall GD, et al. Women with peritoneal carcinomatosis of unknown origin: Efficacy of image-guided biopsy to determine site-specific diagnosis. BJOG 2007;114(01):46-50

42 Wang J, Gao L, Tang S, et al. A retrospective analysis on the diagnostic value of ultrasound-guided percutaneous biopsy for peritoneal lesions. World J Surg Oncol 2013;11:251

43 Daly B, Templeton PA. Real-time CT fluoroscopy: evolution of an interventional tool. Radiology 1999;211(02):309-315

44 Carlson SK, Bender CE, Classic KL, et al. Benefits and safety of CT fluoroscopy in interventional radiologic procedures. Radiology 2001;219(02):515-520

45 Nawfel RD, Judy PF, Silverman SG, Hooton S, Tuncali K, Adams DF. Patient and personnel exposure during CT fluoroscopy-guided interventional procedures. Radiology 2000;216(01):180-184

46 Froelich JJ, Wagner HJ. CT-fluoroscopy: tool or gimmick? Cardiovasc Intervent Radiol 2001;24(05):297-305

47 Stewart CJ, Coldewey J, Stewart IS. Comparison of fine needle aspiration cytology and needle core biopsy in the diagnosis of radiologically detected abdominal lesions. J Clin Pathol 2002;55 (02):93-97

48 Lipnik AJ, Brown DB. Image-guided percutaneous abdominal mass biopsy: technical and clinical considerations. Radiol Clin North Am 2015;53(05):1049-1059

49 Gazelle GS, Haaga JR, Rowland DY. Effect of needle gauge, level of anticoagulation, and target organ on bleeding associated with aspiration biopsy. Work in progress. Radiology 1992;183(02): 509-513

50 Griffin N, Grant LA, Freeman SJ, et al. Image-guided biopsy in patients with suspected ovarian carcinoma: a safe and effective technique? Eur Radiol 2009;19(01):230-235

51 Sainani NI, Arellano RS, Shyn PB, Gervais DA, Mueller PR, Silverman SG. The challenging image-guided abdominal mass biopsy: established and emerging techniques 'if you can see it, you can biopsy it'. Abdom Imaging 2013;38(04):672-696

52 McDermott S, Levis DA, Arellano RS. Approaches to the difficult drainage and biopsy. Semin Intervent Radiol 2012;29(04): 256-263 
53 Arellano RS, Gervais DA, Mueller PR. CT-guided drainage of abdominal abscesses: hydrodissection to create access routes for percutaneous drainage. AJR Am J Roentgenol 2011;196(01):189-191

54 Langen HJ, Jochims M, Günther RW. Artificial displacement of kidneys, spleen, and colon by injection of physiologic saline and $\mathrm{CO}_{2}$ as an aid to percutaneous procedures: experimental results. J Vasc Interv Radiol 1995;6(03):411-416

55 Akins EW, Hawkins IF Jr, Mladinich C, Tupler R, Siragusa RJ, Pry R. The blunt needle: a new percutaneous access device. AJR Am J Roentgenol 1989;152(01):181-182

56 de Bazelaire C, Farges C, Mathieu O, et al. Blunt-tip coaxial introducer: a revisited tool for difficult CT-guided biopsy in the chest and abdomen. AJR Am J Roentgenol 2009;193(02):W144-8

57 Akan H, Ozen N, Incesu L, Gümüş S, Güneş M. Are percutaneous transgastric biopsies using 14-, 16- and 18-G Tru-Cut needles safe? An experimental study in the rabbit. Australas Radiol 1998; 42(02):99-101

58 Tseng HS, Chen CY, Chan WP, Chiang JH. Percutaneous transgastric computed tomography-guided biopsy of the pancreas using large needles. World J Gastroenterol 2009;15(47):5972-5975

$59 \mathrm{Kim}$ JW, Shin SS. Ultrasound-guided percutaneous core needle biopsy of abdominal viscera: tips to ensure safe and effective biopsy. Korean J Radiol 2017;18(02):309-322

60 Maturen KE, Nghiem HV, Marrero JA, et al. Lack of tumor seeding of hepatocellular carcinoma after percutaneous needle biopsy using coaxial cutting needle technique. AJR Am J Roentgenol 2006;187(05):1184-1187

61 Hatfield MK, Beres RA, Sane SS, Zaleski GX. Percutaneous imaging-guided solid organ core needle biopsy: coaxial versus noncoaxial method. AJR Am J Roentgenol 2008;190(02):413-417

62 Gupta S, Ahrar K, Morello FA Jr, Wallace MJ, Madoff DC, Hicks ME. Using a coaxial technique with a curved inner needle for CT- guided fine-needle aspiration biopsy. AJR Am J Roentgenol 2002; 179(01):109-112

63 Singh AK, Leeman J, Shankar S, Ferrucci JT. Core biopsy with curved needle technique. AJR Am J Roentgenol 2008;191(06): 1745-1750

64 Spencer JA, Weston MJ, Saidi SA, Wilkinson N, Hall GD. Clinical utility of image-guided peritoneal and omental biopsy. Nat Rev Clin Oncol 2010;7(11):623-631

65 Vadvala HV, Furtado VF, Kambadakone A, Frenk NE, Mueller PR, Arellano RS. Image-guided percutaneous omental and mesenteric biopsy: assessment of technical success rate and diagnostic yield. J Vasc Interv Radiol 2017;28(11):1569-1576

66 Souza FF, Mortelé KJ, Cibas ES, Erturk SM, Silverman SG. Predictive value of percutaneous imaging-guided biopsy of peritoneal and omental masses: results in 111 patients. AJR Am J Roentgenol 2009;192(01):131-136

67 Huang GT, Sheu JC, Yang PM, Lee HS, Wang TH, Chen DS. Ultrasound-guided cutting biopsy for the diagnosis of hepatocellular carcinoma-a study based on 420 patients. J Hepatol 1996;25(03): 334-338

68 Durand F, Regimbeau JM, Belghiti J, et al. Assessment of the benefits and risks of percutaneous biopsy before surgical resection of hepatocellular carcinoma. J Hepatol 2001;35(02):254-258

69 Chang S, Kim SH, Lim HK, Lee WJ, Choi D, Lim JH. Needle tract implantation after sonographically guided percutaneous biopsy of hepatocellular carcinoma: evaluation of doubling time, frequency, and features on CT. AJR Am J Roentgenol 2005;185(02): 400-405

70 Spencer JA, Swift SE, Wilkinson N, Boon AP, Lane G, Perren TJ. Peritoneal carcinomatosis: image-guided peritoneal core biopsy for tumor type and patient care. Radiology 2001;221(01): $173-177$ 